\title{
Supernova neutrino three-flavor evolution with dominant collective effects
}

\author{
Gianluigi Fogli ${ }^{1,2}$, Eligio Lisi ${ }^{2}$, Antonio Marrone ${ }^{1,2}$ and \\ Irene Tamborra ${ }^{1,2}$ \\ 1 Dipartimento Interateneo di Fisica "Michelangelo Merlin," \\ Via Amendola 173, 70126 Bari, Italy \\ ${ }^{2}$ Istituto Nazionale di Fisica Nucleare, Sezione di Bari, \\ Via Orabona 4, 70126 Bari, Italy
}

\begin{abstract}
Neutrino and antineutrino fluxes from a core-collapse galactic supernova are studied, within a representative three-flavor scenario with inverted mass hierarchy and tiny 1-3 mixing. The initial flavor evolution is dominated by collective selfinteraction effects, which are computed in a full three-family framework along an averaged radial trajectory. During the whole time span considered $(t=1-20 \mathrm{~s})$, neutrino and antineutrino spectral splits emerge as dominant features in the energy domain for the final, observable fluxes. The main results can be useful for SN event rate simulations in specific detectors. Some minor or unobservable three-family features (e.g, related to the muonic-tauonic flavor sector), as well as observable effects due to variations in the spectral input, are also discussed for completeness.
\end{abstract}

PACS numbers: 14.60.Pq, 13.15.+g, 97.60.Bw 


\section{Introduction}

The fluxes of $\nu$ and $\bar{\nu}$ from a core-collapse supernova (SN) can encode very interesting information about both the (anti)neutrino properties [1] and the SN explosion mechanism [2]. Assuming that the latter is understood, one may try to detect distinctive features of flavor change in either the energy $(E)$ or time $(t)$ spectra of the observable fluxes $F_{\alpha}(E, t)$, as compared with the "featureless," unoscillated fluxes $F_{\alpha}^{0}(E, t)$, for one or more neutrino species $\nu_{\alpha}$.

After the recent, seminal work in [3, 4], it has been fully realized that flavor change phenomena driven by (anti)neutrino self-interactions [5] may induce dramatic, observable effects in a variety of SN scenarios, especially for inverted mass hierarchy [6]; see [1, 7] for reviews of this rapidly growing research field. In particular, the socalled spectral split [8] or swap [9] emerges as a distinctive feature in the neutrino [10] and possibly the antineutrino [10, 11] energy spectra. If this feature dominates, then a stepwise flavor conversion of $\nu_{e}$ develops across a certain critical energy $E_{c}$ [12], namely,

$$
P_{e e}(E) \simeq \begin{cases}1 & \left(\text { for } E<E_{c}\right), \\ 0 & \left(\text { for } E>E_{c}\right),\end{cases}
$$

in terms of the survival probability $P_{e e}=P\left(\nu_{e} \rightarrow \nu_{e}\right)$ at the end of collective effects. A somewhat similar feature has been observed in the $\bar{\nu}_{e}$ sector [10, 11], although with at different (generally smaller) split energy $\bar{E}_{c}$,

$$
\bar{P}_{e e}(E) \simeq \begin{cases}1 & \left(E<\bar{E}_{c}\right) \\ 0 & \left(E>\bar{E}_{c}\right) .\end{cases}
$$

In a strictly $2 \nu$ framework with flavors " $e$ " and " $x$ ", the net result of collective effects would be a complete interchange, or "swap," of the fluxes $F_{\alpha}$ between $\left(\nu_{e}, \nu_{x}\right)$ above $E_{c}$ [8, 9, 10], as well as between $\left(\bar{\nu}_{e}, \bar{\nu}_{x}\right)$ above $\bar{E}_{c}$ [11].

Analogously, in a three-flavor scenario with both $\nu_{\mu}$ and $\nu_{\tau}$ acting as a flavor " $x$,"

$$
\begin{aligned}
& 2 F_{x}=F_{\mu}+F_{\tau}, \\
& 2 \bar{F}_{x}=\bar{F}_{\mu}+\bar{F}_{\tau},
\end{aligned}
$$

and with usual initial conditions

$$
F_{\mu}^{0}=F_{\tau}^{0}=F_{x}^{0}=\bar{F}_{x}^{0}=\bar{F}_{\tau}^{0}=\bar{F}_{\mu}^{0}
$$

a swap is expected to occur between the $e$ flavor and one of the two $x$ flavors, namely,

$$
\begin{aligned}
& F_{e}^{\prime}=F_{e}^{0} P_{e e}+F_{x}^{0}\left(1-P_{e e}\right) \simeq \begin{cases}F_{e}^{0} & \left(E<E_{c}\right), \\
F_{x}^{0} & \left(E>E_{c}\right),\end{cases} \\
& \bar{F}_{e}^{\prime}=\bar{F}_{e}^{0} \bar{P}_{e e}+\bar{F}_{x}^{0}\left(1-\bar{P}_{e e}\right) \simeq \begin{cases}\bar{F}_{e}^{0} & \left(E<\bar{E}_{c}\right), \\
\bar{F}_{x}^{0} & \left(E>\bar{E}_{c}\right),\end{cases} \\
& 2 F_{x}^{\prime}=\left[F_{e}^{0}\left(1-P_{e e}\right)+F_{x}^{0} P_{e e}\right]+F_{x}^{0} \simeq \begin{cases}2 F_{x}^{0} & \left(E<E_{c}\right), \\
F_{e}^{0}+F_{x}^{0} & \left(E>E_{c}\right),\end{cases} \\
& 2 \bar{F}_{x}^{\prime}=\left[\bar{F}_{e}^{0}\left(1-\bar{P}_{e e}\right)+\bar{F}_{x}^{0} \bar{P}_{e e}\right]+\bar{F}_{x}^{0} \simeq \begin{cases}2 \bar{F}_{x}^{0} & \left(E<\bar{E}_{c}\right), \\
\bar{F}_{e}^{0}+\bar{F}_{x}^{0} & \left(E>E_{c}\right) .\end{cases}
\end{aligned}
$$


In the above equations, the primes denote fluxes at the end of collective effects within the SN, to be further evolved up to the exit from the SN and to the final detector.

It is important to test the above $3 \nu$ expectations within comprehensive three-flavor calculations, for various reasons. First, one has to show that the assumed dominance of spectral split phenomena can indeed be manifest (without being disrupted by ordinary matter effects) in a sufficiently general, uncontrived and interesting supernova $3 \nu$ scenario. Second, the effective factorization of one family out of three self-interacting families (leading to nonlinear equations) is never totally obvious, and "effective $2 \nu$ " expectations like those in Eqs. (66) -(9) need to be explicitly checked. Third, in the selfinteraction context, the probabilities $P_{\alpha \beta}$ depend, among other things, on the initial conditions and on the absolute fluxes - which do change if the SN energy luminosity (which also varies in time) is distributed over $3 \nu$ rather than $2 \nu$. Finally, while the $\nu$ spectral split phenomenon is robust and largely understood in terms of lepton number conservation and adiabatic flavor evolution [13, 14], the $\bar{\nu}$ split seems to be more fragile and related to (not completely understood) nonadiabatic aspects of the evolution [11]; it is thus worth checking its appearance in a full $3 \nu$ calculation.

Several recent works have focussed on collective effects in $3 \nu$ scenarios [15, 16, 17, $18,19,20,21,22$ and have successfully recovered spectral split features in the neutrino sector (and perhaps also in the antineutrino sector [17, 22]) in inverted hierarchy. We think it useful to add an independent contribution to this very recent research field, by discussing a SN $3 \nu$ scenario where both the $\nu$ and $\bar{\nu}$ split features are shown to emerge clearly for a relatively long time of $\sim 20$ seconds after SN explosion. Such a scenario is thus particularly suited to prospective experimental tests in high-statistics, time-integrated energy spectra of events from the next galactic core-collapse SN.

Our work is structured as follows. In Sec. 2 we describe a representative SN neutrino framework, where collective effects (in the form of spectral splits) are expected to dominate over ordinary matter effects. In Sec. 3 we discuss the $3 \nu$ formalism used to compute the flavor evolution of the initial fluxes $F_{\alpha}^{0}$. In Sec. 4 we present our results for the fluxes $F_{\alpha}^{\prime}$ at the end of collective effects, and show that they confirm the simple expectations in Eqs. (6) -(9); we also discuss minor or unobservable features of our calculations. In Sec. 5 we complete the flavor evolution of the observable fluxes $F_{\alpha}$, by accounting for final phase-averaging and matter effects. In Sec. 6 we discuss the effects of some variations in the input SN neutrino spectra, with respect to our default scenario. Section 7 summarizes our results.

The reader is reminded that our results, as well as many other observable features discussed in the growing literature of self-interaction neutrino effects in supernovae, must be taken with a grain of salt. Indeed, many open questions are still open from the viewpoint of the theory (validity of the mean-field approximation in the neutrino evolution equations), of the SN explosion energetics (neutrino luminosities and energy spectra) and geometry (asymmetries, turbulence), and of the neutrino evolution numerics (convergence and robustness of calculations). Results which currently appear to be rather generic (such as the spectral split phenomena in inverse hierarchy) might 
be unpredictably challenged by improvements and further understanding in any of the above issues. Hopefully, the next galactic supernova explosion(s) will help to reduce the current level of uncertainty in the physics and astrophysics hidden in the expected neutrino signal.

\section{Reference neutrino parameters and SN scenario}

The adopted $3 \nu$ oscillation parameters and our reference SN model are discussed below.

\subsection{Neutrino parameters}

We assume that the hierarchy of neutrino masses $m_{i}$ is inverted $\left(m_{3}<m_{1,2}\right)$,

$$
\left(m_{1}^{2}, m_{2}^{2}, m_{3}^{2}\right)=\frac{m_{1}^{2}+m_{2}^{2}}{2}+\left(-\frac{\delta m^{2}}{2},+\frac{\delta m^{2}}{2},-\Delta m^{2}\right),
$$

with squared mass splittings set at the representative values

$$
\begin{aligned}
& \Delta m^{2}=2 \times 10^{-3} \mathrm{eV}^{2}, \\
& \delta m^{2}=8 \times 10^{-5} \mathrm{eV}^{2} .
\end{aligned}
$$

The associated "high" $(H)$ and "low" $(L)$ vacuum oscillation frequencies are then

$$
\begin{aligned}
& \omega_{H}=\frac{\Delta m^{2}}{2 E}=\frac{5.07}{E / \mathrm{MeV}}\left[\mathrm{km}^{-1}\right], \\
& \omega_{L}=\frac{\delta m^{2}}{2 E}=\frac{0.20}{E / \mathrm{MeV}}\left[\mathrm{km}^{-1}\right] .
\end{aligned}
$$

Within the usual parametrization [23] for the mixing matrix $U=$ $U\left(\theta_{12}, \theta_{13}, \theta_{23}, \delta_{\mathrm{CP}}\right)$, we fix $\theta_{12}$ as 24

$$
\sin ^{2} \theta_{12}=0.314
$$

while we consider three representative values for the more uncertain angle $\theta_{23}$, corresponding to maximal and nonmaximal (but octant-symmetric) mixing:

$$
\sin ^{2} \theta_{23}=0.50,0.36,0.64 \text {. }
$$

Concerning the third angle $\theta_{13}$, we assume a tiny reference value,

$$
\sin ^{2} \theta_{13}=10^{-6}
$$

in order to suppress the impact of ordinary matter effects, as explained in the following Subsection 2.2. Finally, we ignore possible CP-violating effects (which are arguably very small [22]) by setting

$$
\delta_{\mathrm{CP}}=0 .
$$



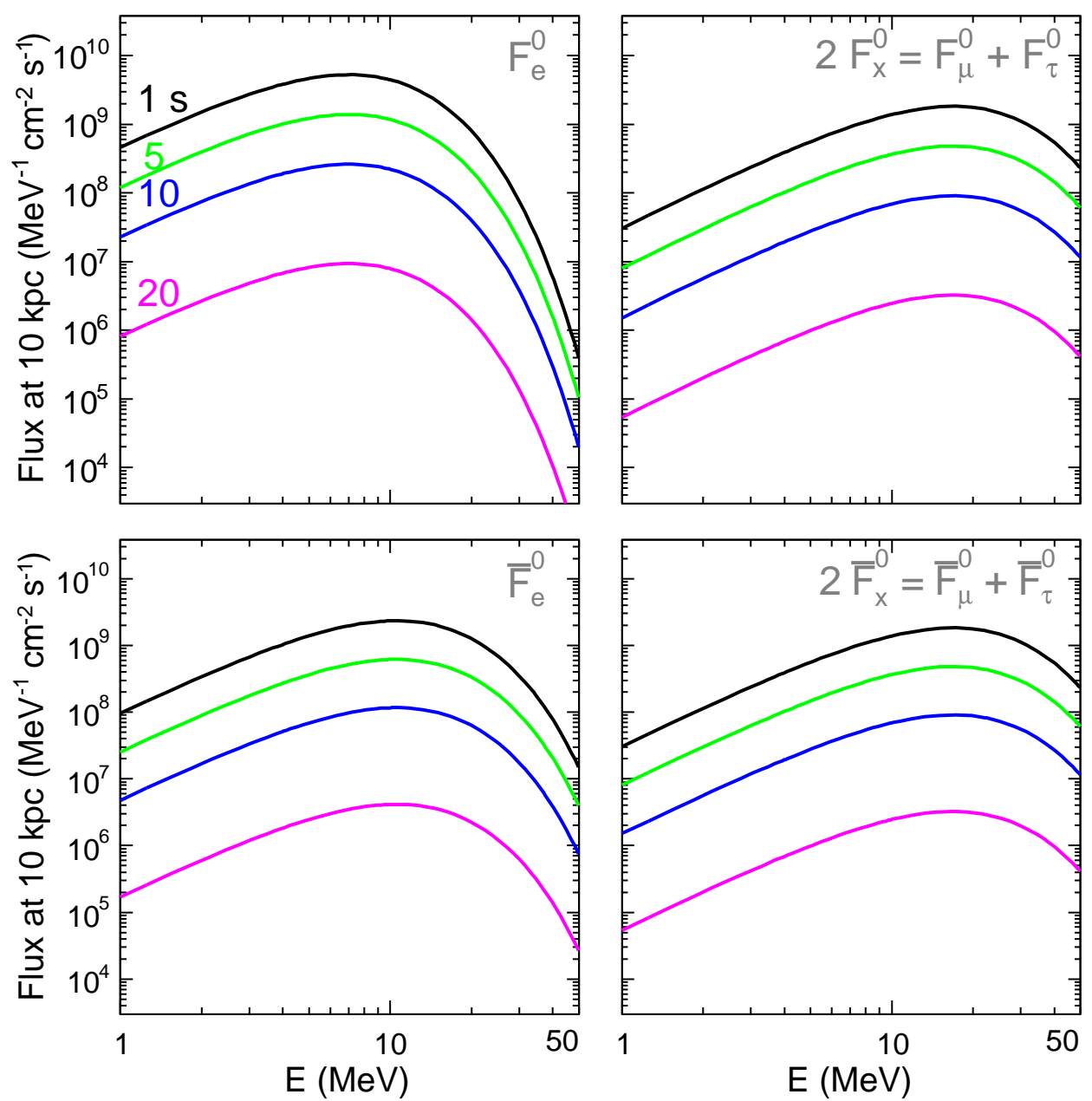

Figure 1. Unoscillated fluxes of neutrinos $\left(F_{\alpha}^{0}\right.$, top) and antineutrinos $\left(\bar{F}_{\alpha}^{0}\right.$, bottom) with electron flavor (left) and $x$ flavor (right). In the latter case, we plot the sum of muonic and tauonic fluxes. All fluxes refer to our reference supernova model and to a distance $d=10 \mathrm{kpc}$. The black, green, blue and magenta curves correspond to $t=1$, 5,10 , and $20 \mathrm{~s}$, respectively.

\subsection{Supernova model}

Our reference SN scenario is essentially taken from [25], with minor changes in the average neutrino energies. We assume a galactic core-collapse supernova releasing a binding energy $E_{B}=3 \times 10^{53} \mathrm{erg}$, equally distributed among the six $(3 \nu+3 \bar{\nu})$ species, and distributed in time with a time constant $\tau=3 \mathrm{~s}$. The energy luminosity associated to each species is thus

$$
L(t)=\frac{E_{B}}{6} \frac{e^{-t / \tau}}{\tau} .
$$

At the conventional distance for a galactic supernova,

$$
d=10 \mathrm{kpc},
$$


the unoscillated flux of the neutrino species $\nu_{\alpha}$, per unit of area, time, and energy, is expected to be

$$
F_{\alpha}^{0}(E, t)=\frac{L(t)}{4 \pi d^{2}} \frac{\phi_{\alpha}^{0}(E)}{\left\langle E_{\alpha}\right\rangle}
$$

where we assume normalized thermal energy spectra $\phi_{\alpha}^{0}(E)$ with average energy $\left\langle E_{\alpha}\right\rangle$. As in [10], inspired by [26], we set

$$
\left\langle E_{e}\right\rangle=10 \mathrm{MeV},\left\langle\bar{E}_{e}\right\rangle=15 \mathrm{MeV},\left\langle E_{x}\right\rangle=24 \mathrm{MeV},
$$

corresponding to temperatures $\left(T_{e}, T_{\bar{e}}, T_{x}\right)=(3.17,4.76,7.62) \mathrm{MeV} \ddagger$ Figure 1 shows the corresponding fluxes $F_{\alpha}^{0}(E, t)$ in the energy interval $E \in[1,50] \mathrm{MeV}$, at four representative times $(t=1,5,10$, and $20 \mathrm{~s})$.

Concerning the SN geometry, we adopt the spherically-symmetric bulb model [4], with a neutrinosphere radius $R_{\nu}=10 \mathrm{~km}$. Within this model, the previous initial conditions for the neutrino luminosity and unoscillated fluxes define, at any radius $r>R_{\nu}$, the effective density of neutrinos $\left(N=N_{e}+N_{\mu}+N_{\tau}\right)$ and of antineutrinos $(\bar{N})$ per unit volume, as well as the self-interaction potential

$$
\mu(r)=\sqrt{2} G_{F}[N(r)+\bar{N}(r)] .
$$

For each species $\nu_{\alpha}$, it is useful to introduce also density $n_{\alpha}$ per unit of volume and energy (see [4, 10] for details):

$$
N_{\alpha}=\int d E n_{\alpha}(E) \text {. }
$$

Figure 2 (left panel) shows the function $\mu(r)$, up to $r=500 \mathrm{~km}$, for the four $t$ values chosen. In the same panel, the shaded horizontal band corresponds to the range $\mu \in\left[\mu_{\text {inf }}, \mu_{\text {sup }}\right] \simeq[6.7,67] \mathrm{km}^{-1}$ where, according to the estimate in [10], collective bipolar [27] ("pendulum" [6]) oscillations are expected to develop (after a "synchronized" regime [27, 6, 28]) for this $\mathrm{SN}$ scenario in the $2 \nu$ case. We have explicitly verified that these expectations also hold in our full $3 \nu$ flavor evolution, with a radial accuracy better than $\sim 10 \mathrm{~km}$ (not shown). For example, at $t=10 \mathrm{~s}$, we find numerically a bipolar development range $r \simeq[60,100] \mathrm{km}$, in good agreement with the range $r \simeq[55,95] \mathrm{km}$ derived from the intersection of the horizontal band with the $\mu(r)$ curve at $t=10 \mathrm{~s}$.

However, collective phenomena extend somewhat beyond the bipolar range, and eventually vanish when the spectral splits are complete. As a rule of thumb, the end of all collective effects occurs at a radius $r_{\text {end }}$ where the self-interaction potential $\mu$ equals a relatively small value $\mu_{\text {end }}$. In our numerical experiments, we find that $\mu_{\text {end }} \simeq 0.5 \mathrm{~km}^{-1}$ provides a reasonable estimate for the radius $r_{\text {end }}$

$$
\mu\left(r_{\text {end }}\right) \simeq 0.5 \mathrm{~km}^{-1}
$$

at any time $t$ (e.g., $r_{\text {end }} \simeq 400 \mathrm{~km}$ at $t=1 \mathrm{~s}$ in Fig. 2). For safety, we have always checked that our results do not appreciably change by continuing the numerical flavor evolution for further $\sim 100 \mathrm{~km}$ or more (e.g., up to $r \simeq 500 \mathrm{~km}$ for $t=1 \mathrm{~s}$ ).

$\ddagger$ Effects of variations with respect to the default choice in Eq. (22) will be discussed in Sec. 6 . 

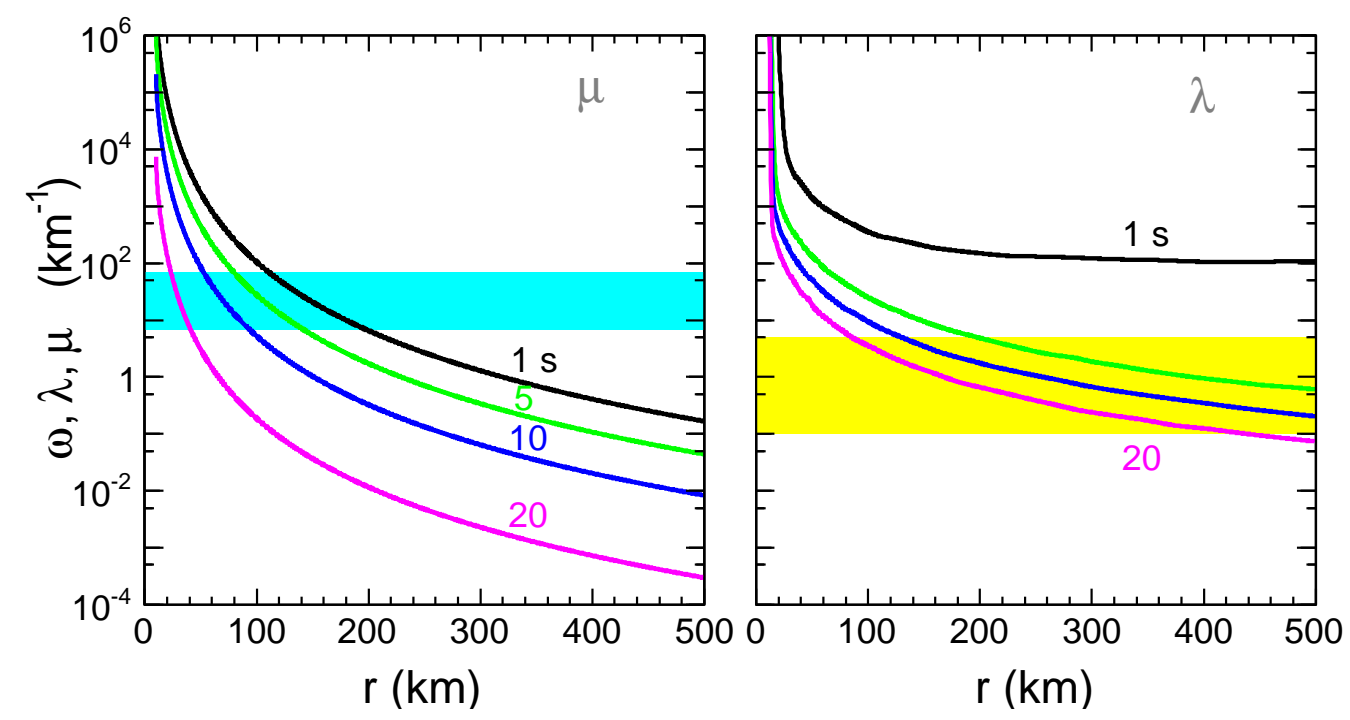

Figure 2. Radial profiles of the self-interaction potential $\mu$ (left) and of the matter potential $\lambda$ (right). The black, green, blue and magenta curves correspond to $t=1$, 5,10 , and $20 \mathrm{~s}$, respectively. In the left panel, the shaded horizontal band marks the $\mu$ range where bipolar effects develop. In the right panel, the shaded band marks the range of the high vacuum frequency $\omega_{H}$ for $E \in[1,50] \mathrm{MeV}$, where MSW effects may develop if the $H$-resonance condition $\left(\lambda \sim \omega_{H}\right)$ is satisfied.

Figure 2 (right panel) shows the radial profile of the matter potential $\lambda$ at different times, as taken from [25]. As usual, the matter potential is defined as

$$
\lambda(r)=\sqrt{2} G_{F} N_{e^{-}}(r),
$$

where $N_{e^{-}}(r)$ is the net electron density. In inverted hierarchy, antineutrinos may undergo significant matter effects (or MSW effects, from Mikheev, Smirnov, and Wolfenstein [29]) when the so-called $H$-resonance condition is fulfilled (see, e.g., [30]),

$$
\lambda \simeq \omega_{H} .
$$

For the energy range in Fig. $1(E \in[1,50] \mathrm{MeV})$, the relevant interval for the high vacuum frequency, $\omega_{H} \in[0.1,5] \mathrm{km}^{-1}$, is shown in the right panel of Fig. 2 as a shaded horizontal band. [Note in Fig. 2 that the $\omega_{H}$ value of $0.5 \mathrm{~km}^{-1}$ in Eq. (25) is in the middle of such band.] Its intersection with one of the $\lambda(r)$ curves marks the related $H$-resonance range (for example, from Fig. $2, r \gtrsim 130 \mathrm{~km}$ at $t=10 \mathrm{~s}$ ).

A comparison of the left and right panels in Fig. 2 shows that, in our reference SN model, collective bipolar oscillations develop before possible $H$-resonance effects at any time (while they may be coupled in other scenarios with shallower matter profiles, see e.g. [4, 19]). Flavor swap effects, however, extend up to $r_{\text {end }}$ estimated previously, and may partly enter the $H$-resonance region for large $\omega_{H}$ at $t>1$. However, for values of $\theta_{13}$ (at least) as small as in Eq. (17), the $H$ resonance is highly nonadiabatic, and produces no further flavor conversion at any relevant SN energy [30]. We have numerically verified the absence of MSW flavor conversion for antineutrinos in inverted hierarchy (besides 
their collective flavor swap) for $r$ well within the $H$-resonance region. We have also verified that all our main results in Secs. 4 and 5 remain unchanged for reasonably smaller values of $\sin ^{2} \theta_{13}$ (e.g., $10^{-7}$ or $10^{-8}$ ), which simply cause a logarithmic delay of the bipolar oscillation onset [3, 6]. As an added bonus, for $\sin ^{2} \theta_{13} \lesssim 10^{-6}$, one gets a basically complete suppression of: $(i)$ possible matter effects due to shock-wave features (see, e.g., [31]); (ii) possible decoherence effects due to density fluctuations (see, e.g., [32]); (iii) Earth matter effects, if any, before detection (see, e.g., [1, 20]). These welcome simplifications make neutrino-neutrino interaction effects dominate over neutrino-matter interaction effects at any time $t \geq 1 \mathrm{~s}$ in our framework, and motivate a posteriori our choice for the tiny mixing angle in Eq. (17).

A final remark is in order. As described in the next Sections, we evolve the neutrino flavors along a single, averaged radial trajectory. This (so-called "single-angle") approximation is often reasonable - when compared with few available "multi-angle" calculations - but it may fail badly when the matter potential is so high that its gradient depends sensitively on the trajectory (see [33], and references therein). For our inspiring SN model [25], this situation may occur only at very early stages $(t<1 \mathrm{~s})$, which are excluded from the present investigation.

\section{Three-flavor formalism}

The density matrix formalism is particularly useful to deal with neutrino selfinteractions, which depend on the neutrino density itself. In the two-family case, the traceless part of the $(2 \times 2)$ density matrix $\boldsymbol{\rho}$ can be expanded onto Pauli matrices $\sigma_{i}$, with projections coefficients forming a 3 -vector $\mathbf{P}$ for each energy mode $E$ [6].

In the three-family case, we have generalized the $2 \nu$ evolution equations to the $3 \nu$ case [34, by projecting the $(3 \times 3)$ density matrix $\boldsymbol{\rho}$ in flavor basis onto Gell-Mann matrices $\lambda_{i}$, in terms of an 8-component vector $\mathbf{P}$. The resulting equations 34 are formally similar to those recently discussed in [17, 18, 19], and are briefly reviewed below for completeness.

Given an 8-dimensional orthonormal basis $\left(\mathbf{e}_{1}, \ldots, \mathbf{e}_{8}\right)$ for the $\mathbf{P}$ vector space,

the flavor projector matrices, $\boldsymbol{\Pi}_{e}=\operatorname{diag}(1,0,0), \boldsymbol{\Pi}_{\mu}=\operatorname{diag}(0,1,0)$ and $\boldsymbol{\Pi}_{\tau}=$ $\operatorname{diag}(0,0,1)$, can be represented as

$$
\boldsymbol{\Pi}_{\alpha}=\frac{\mathbf{1}}{3}+\mathbf{u}_{\alpha} \cdot \frac{\boldsymbol{\lambda}}{2}(\alpha=e, \mu, \tau)
$$

where 1 is the $3 \times 3$ unit matrix, $\boldsymbol{\lambda}=\sum \lambda_{i} \mathbf{e}_{i}$, and the $\lambda_{i}$ 's are the Gell-Mann matrices with usual conventions: $\lambda_{i}=\lambda_{i}^{\dagger} ; \operatorname{tr}\left(\lambda_{i}\right)=0 ; \operatorname{tr}\left(\lambda_{i} \lambda_{j}\right)=2 \delta_{i j}$; and $\left[\lambda_{i}, \lambda_{j}\right]=2 i f_{i j k} \lambda_{k}$. The $\mathbf{u}_{\alpha}$ vectors (with $\left|\mathbf{u}_{\alpha}\right|=2 / \sqrt{3}$ ) read:

$$
\mathbf{u}_{e}=\mathbf{e}_{3}+\frac{1}{\sqrt{3}} \mathbf{e}_{8}, \mathbf{u}_{\mu}=-\mathbf{e}_{3}+\frac{1}{\sqrt{3}} \mathbf{e}_{8}, \mathbf{u}_{\tau}=-\frac{2}{\sqrt{3}} \mathbf{e}_{8} .
$$

The neutrino density matrix, at given energy $E$, is projected via

$$
\boldsymbol{\rho}=n\left(\frac{1}{3}+\mathbf{P} \cdot \frac{\boldsymbol{\lambda}}{2}\right)
$$


where $\mathbf{P}=\sum P_{i} \mathbf{e}_{i}$ (with $|\mathbf{P}|=2 / \sqrt{3}$ ), and $n$ is the neutrino density per unit volume and energy introduced before. At any time $t$ and energy $E$, the $\nu_{\alpha}$ content is given by $\operatorname{tr}\left(\boldsymbol{\rho}(t) \boldsymbol{\Pi}_{\alpha}\right)$.

The evolution of $\boldsymbol{\rho}$ is governed by the Liouville equation $i \dot{\boldsymbol{\rho}}=[\mathbf{H}, \boldsymbol{\rho}]$, where the Hamiltonian $\mathbf{H}$ contains vacuum, matter, and self-interaction terms. In the vacuum term, we include both low and high vacuum frequencies ( $\omega_{L}$ and $\omega_{H}$, respectively), and assume inverted hierarchy. In the matter term, we include the $\nu_{\tau}-\nu_{\mu}$ potential difference at one loop [35], whose size is $\delta \lambda / \lambda \simeq 5 \times 10^{-5}$ (see also [16, 18]). By expanding $\boldsymbol{\rho}$ and $\mathbf{H}$ onto Gell-Mann matrices, evolution equations are obtained for the $\nu$ flavor polarization vector $\mathbf{P}$ and, analogously, for the $\bar{\nu}$ vector $\overline{\mathbf{P}}$ :

$$
\begin{aligned}
& \dot{\mathbf{P}}=\left[+\left(\omega_{L} \mathbf{B}_{L}-\omega_{H} \mathbf{B}_{H}\right)+\lambda \mathbf{v}+\mu \mathbf{D}\right] \times \mathbf{P}, \\
& \dot{\mathbf{P}}=\left[-\left(\omega_{L} \mathbf{B}_{L}-\omega_{H} \mathbf{B}_{H}\right)+\lambda \mathbf{v}+\mu \mathbf{D}\right] \times \overline{\mathbf{P}},
\end{aligned}
$$

where the 8-component vector product is defined as $(\mathbf{a} \times \mathbf{b})_{i}=f_{i j k} a_{j} b_{k}$.

In the above equations, the first (vacuum) terms embed the squared mass splittings via $\omega_{L, H}$, and the mixing angles via effective "magnetic fields" $\mathbf{B}_{L, H}$ with components

$$
\mathbf{B}_{L}=\left(\begin{array}{c}
c_{13}\left(S_{12} c_{23}+C_{12} s_{13} s_{23}\right) \\
0 \\
-\frac{1}{8} C_{12}\left(3+3 C_{13}+3 C_{23}-C_{13} C_{23}\right)+\frac{1}{2} s_{13} S_{12} S_{23} \\
\frac{1}{2} C_{12} S_{13} c_{23}-S_{12} c_{13} s_{23} \\
0 \\
-s_{13} C_{23} S_{12}-\frac{1}{4}\left(3-C_{13}\right) S_{23} C_{12} \\
0 \\
-\frac{\sqrt{3}}{8} C_{12}\left(1+C_{13}-3 C_{23}+C_{13} C_{23}\right)-\frac{\sqrt{3}}{2} s_{13} S_{12} S_{23}
\end{array}\right),
$$

and

$$
\mathbf{B}_{H}=\left(\begin{array}{c}
S_{13} s_{23} \\
0 \\
\frac{1}{4}\left(1-3 C_{13}+C_{23}+C_{13} C_{23}\right) \\
S_{13} c_{23} \\
0 \\
\frac{1}{2} S_{23}\left(1+C_{13}\right) \\
0 \\
\frac{1}{4 \sqrt{3}}\left(1-3 C_{13}-3 C_{23}-3 C_{13} C_{23}\right)
\end{array}\right)
$$

where we have set $s_{i j}=\sin \theta_{i j}, c_{i j}=\cos \theta_{i j}, S_{i j}=\sin 2 \theta_{i j}$, and $C_{i j}=\cos 2 \theta_{i j}$.

The second (matter interaction) term $\lambda \mathbf{v}$ in Eqs. (31) and (32) embeds the $\nu_{e}-\nu_{\mu, \tau}$ interaction energy difference (oriented along $\mathbf{u}_{e}$ ), as well as the tiny correction due the $\nu_{\tau}-\nu_{\mu}$ interaction energy difference (oriented along $\mathbf{u}_{\tau}$ ),

$$
\lambda \mathbf{v}=\lambda\left(\mathbf{u}_{e}+\frac{\delta \lambda}{\lambda} \mathbf{u}_{\tau}\right) .
$$


The third (self-interaction) term $\mu \mathbf{D}$ couples all neutrino and antineutrino modes via the integral vector difference

$$
\mathbf{D}=\frac{1}{N+\bar{N}} \int d E(n \mathbf{P}-\bar{n} \overline{\mathbf{P}}) .
$$

Note that, in general, neutrino self-interactions depend on the crossing angle between the neutrino trajectories [5, 4. We have implicitly assumed that such dependence can be averaged out along a single, radial trajectory (single-angle approximation, see [10] and references therein). If crossing angles were accounted for, the equations would entail a further angular variable (multi-angle description) [4, 7, 10, 36] not included in the present investigation.

We discretize in energy the coupled evolution equations (31) and (321), and solve them by numerical integration (see [10] for details), up to the end of collective effects $\left(r_{\text {end }} \lesssim 500 \mathrm{~km}\right)$. The initial conditions are fixed by the SN model described in the previous Section, for each representative time: $t=1,5,10$ and $20 \mathrm{~s}$. The results are described below in the graphically convenient range $E \in[1,50] \mathrm{MeV}$ (although the numerical evolution includes modes with $E<1 \mathrm{MeV}$ ).

\section{Intermediate fluxes after collective effects}

In this Section we discuss our numerical results for the intermediate fluxes $F_{\alpha}^{\prime}$ at the end of collective effects $\left(r=r_{\text {end }} \lesssim 500 \mathrm{~km}\right.$ ), and show that they confirm the expectations in Eqs. (6) - (9). In order to allow a visual comparison with the unoscillated fluxes $F_{\alpha}^{0}$ at $d=10 \mathrm{kpc}$ (Fig. 1), the fluxes $F_{\alpha}^{\prime}$ are rescaled by a factor $\left(r_{\text {end }} / d\right)^{2}$. We stress that the intermediate fluxes $F_{\alpha}^{\prime}$ are unobservable, as both $\nu$ and $\bar{\nu}$ are subject to further flavor evolution in the SN, and to phase-averaging effects up to the detector. The final, observable fluxes $F_{\alpha}$ at $d=10 \mathrm{kpc}$ will be estimated in the next Section.

Figure 3 shows our computed fluxes $F_{\alpha}^{\prime}$, in the same scale as Fig. 1. The comparison of Figs. 1 and 3 confirms that spectral splits of neutrinos and antineutrinos emerge as dominant features, as expected. In our adopted scenario, the neutrino split occurs around a critical energy $E_{c} \simeq 7 \mathrm{MeV}[10$, dictated by lepton number conservation [13, 14. The antineutrino split occurs at a somewhat lower energy $\bar{E}_{c} \simeq$ few $\mathrm{MeV}$, which is not predicted a priori so far [11]. However, apart from a transition region (about 1-2 MeV wide) around $E_{c}$ and $\bar{E}_{c}$, the evolved fluxes in Fig. 3 coincide with those expected from the application of Eqs. (6) - (9) to the unevolved fluxes of Fig. 1.

This is shown in more detail in Fig. 4 , with reference to the time $t=10 \mathrm{~s}$ (similar results hold at 1, 5, and $20 \mathrm{~s}$ ). Below the critical energy, all $\nu_{\alpha}$ species remain unchanged: the evolved fluxes $F_{\alpha}^{\prime}$ (solid blue curves) coincide with the unevolved fluxes $F_{\alpha}^{0}$ (dashed blue curves) in the upper left panel. Conversely, above the critical energy, the electron flavor fully converts. As a consequence, in the lower left panels of Fig. 4, the evolved fluxes $F_{e}^{\prime}$ and $\bar{F}_{e}^{\prime}$ turn into $F_{x}^{0}$ and $\bar{F}_{x}^{0}$, respectively (dashed red curves). Antineutrinos show a similar behavior (right panels). Therefore, Eqs. (6) -(9) hold with good accuracy, 

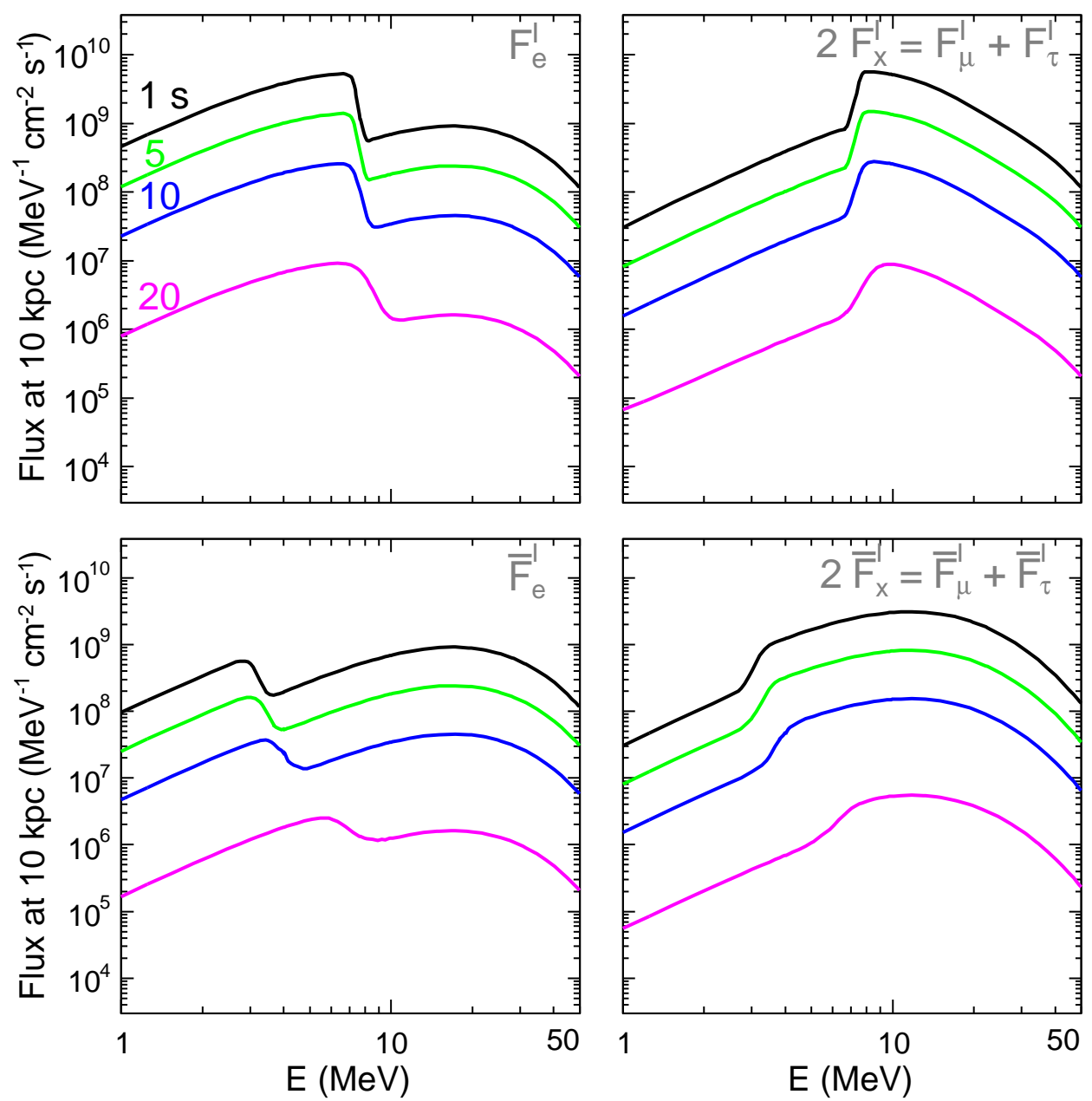

Figure 3. Fluxes of neutrinos $\left(F_{\alpha}^{\prime}\right)$ and antineutrinos $\left(\bar{F}_{\alpha}^{\prime}\right)$ at the end of collective effects, rescaled to $d=10 \mathrm{kpc}$ for comparison with Fig. 1. See the text for details.

except close to the spectrum step, whose details may also depend on the adopted singleangle approximation. Indeed, multi-angle calculations (not performed in this work) may further widen the transition region [10], especially for antineutrinos [11. However, they are not expected to change the low- and high-energy limits of the spectra.

The above $3 \nu$ results, obtained at the end of collective effects, are not significantly influenced by the subdominant "solar" squared mass difference $\left(\delta m^{2}\right)$ or by the $\nu_{\mu}-\nu_{\tau}$ interaction energy difference $(\delta \lambda)$. Effects of $\delta m^{2}$ are expected to be larger at relatively shallow matter densities [18, 19], i.e., at larger $t$ in Fig. 2; we find the largest fractional flux variations at $t=20 \mathrm{~s}$, as obtained by setting $\delta m^{2}=0$, to be negliglible ( $<$ few $\%$ ). Conversely, effects of $\delta \lambda$ are expected to be larger at higher matter densities [16, 18, 22], i.e., at shorter $t$; we find the largest variations at $t=1 \mathrm{~s}$, as obtained by setting $\delta \lambda / \lambda=0$, to be also negligible $(<$ few $\%)$. We find that these variations are mainly localized around the critical split energies, and can thus be mainly attributed to small, subleading $3 \nu$ collective effects.

To a very good accuracy, our results are independent of any effect which may change 

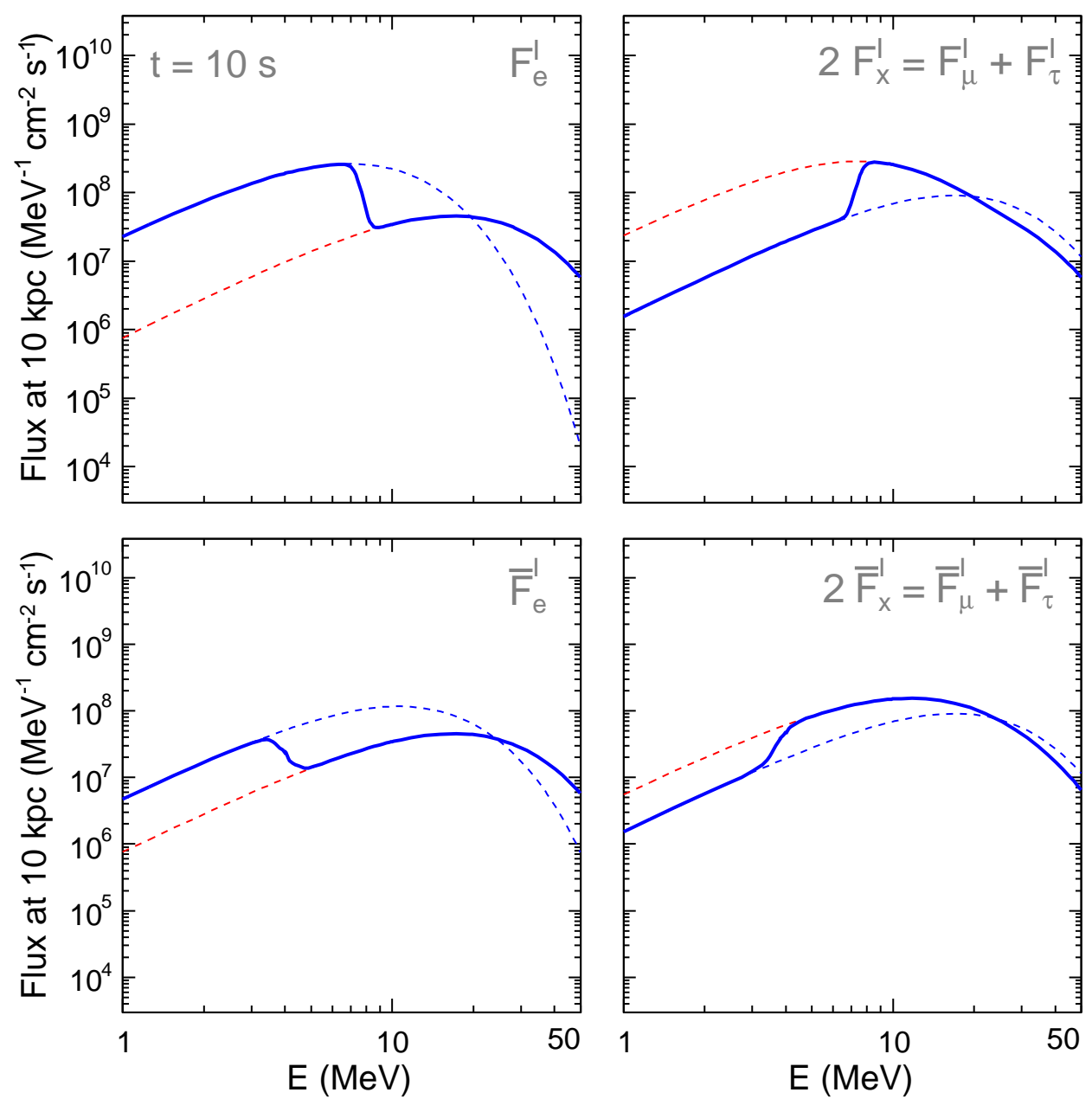

Figure 4. Fluxes of neutrinos and antineutrinos at the end of collective effects, for $t=10 \mathrm{~s}$. Solid blue curves: computed fluxes $F_{\alpha}^{\prime}$. Dashed blue and red curves: limiting behavior at low-energy and high-energy, respectively, in terms of unoscillated fluxes $F_{\alpha}^{0}$, according to Eqs. (6) (9).

the relative $\nu_{\mu}$ and $\nu_{\tau}$ flux proportions within the sum $2 F_{x}^{\prime}=F_{e}^{\prime}+F_{\mu}^{\prime}$, including not only the small $\nu_{\mu, \tau}$ correction $\delta \lambda / \lambda$, but also the (quasi)maximal $\nu_{\mu, \tau}$ mixing angle $\theta_{23}$. Indeed, the curves in Fig. 3 do not depend on the chosen value of $\sin ^{2} \theta_{23}$ in Eq. (16), as we have explicitly checked.

For instance, Fig. 5 shows both the absolute fluxes with $e$ and $x$ flavor (upper panels) and the muonic-to-tauonic flavor ratio (lower panels) at the end of collective effects, for $t=10 \mathrm{~s}$; similar results hold at different $t$ (not shown). The curves in the upper panels do not depend on the indicated value of $\sin ^{2} \theta_{23}$, while those in the lower one do. More precisely, for neutrinos (lower left panel) the ratio $F_{\mu}^{\prime} / F_{\tau}^{\prime}$ remains equal to $1\left(=F_{\mu}^{0} / F_{\tau}^{0}\right)$ in three cases: $(i)$ at maximal mixing, $\theta_{23}=\pi / 4$, where $\nu_{\mu}$ and $\nu_{\tau}$ are interchangeable, up to minor $\delta \lambda / \lambda$ effects; (ii) below the critical energy $E_{c} \simeq 7 \mathrm{MeV}$, where there is no net flavor conversion; and (iii) at the specific "equalization" energy $E_{\text {eq }} \simeq 20 \mathrm{MeV}$ where $F_{e}^{0}=F_{x}^{0}$, and flavor conversions become inoperative: $F_{e}^{\prime}=F_{x}^{\prime}$. Similarly for 

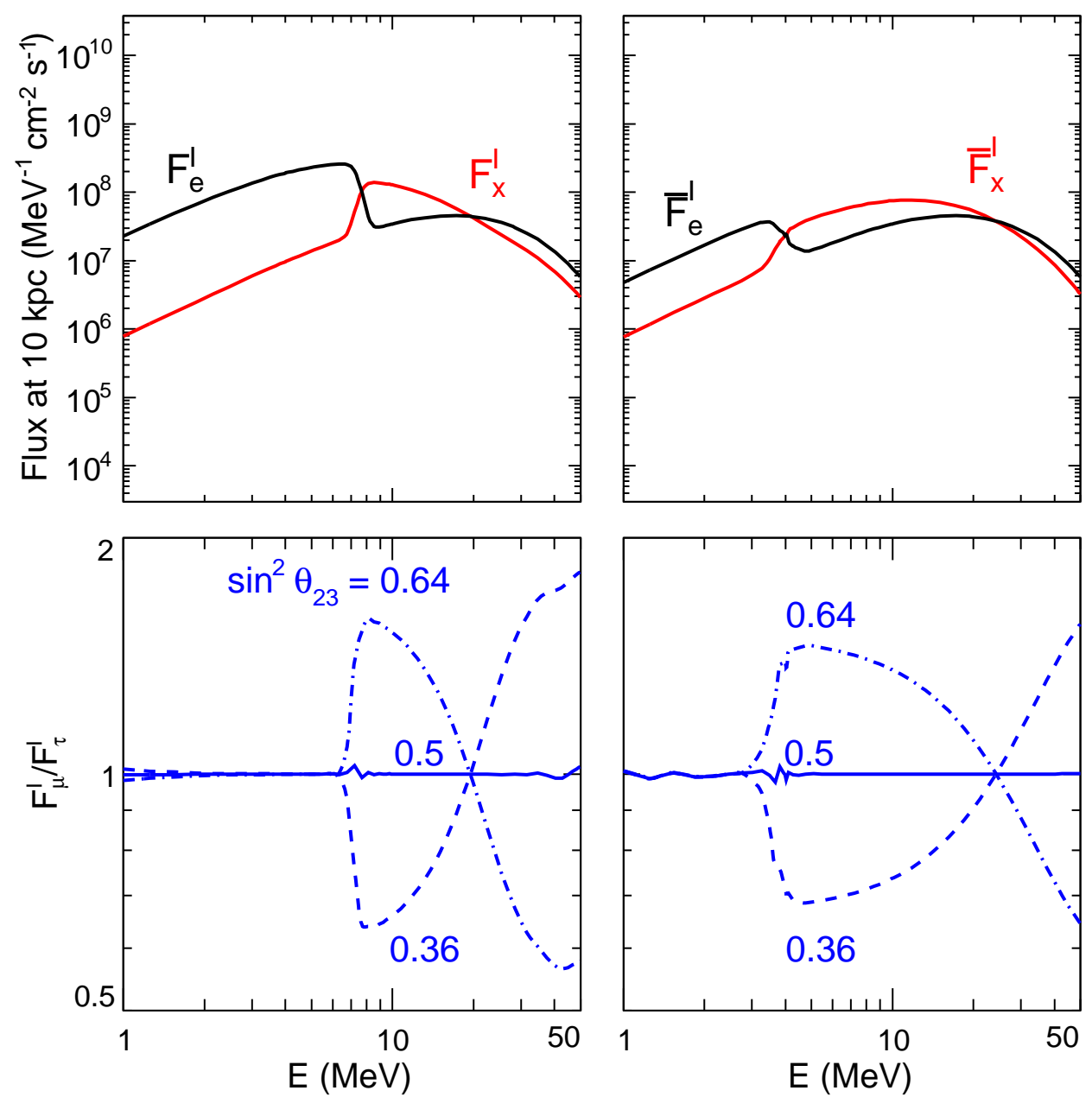

Figure 5. Neutrino and antineutrino fluxes $F_{\alpha}^{\prime}$ at the end of collective effects $(r \lesssim 500 \mathrm{~km})$, for $t=10 \mathrm{~s}$. Upper panels: absolute fluxes. Lower panels: muonicto-tauonic flux ratio for $\nu$ (left) and $\bar{\nu}$ (right), for $\sin ^{2} \theta_{23}=0.36,0.5,0.64$.

antineutrinos (lower right panel), but with $\bar{E}_{c} \simeq 3-4 \mathrm{MeV}$ and $\bar{E}_{\text {eq }} \simeq 27 \mathrm{MeV}$.

In Fig. 5 , the cases where $F_{\mu}^{\prime} / F_{\tau}^{\prime} \neq 1$ can be understood by considering that, in inverted hierarchy, flavor conversions occur mainly between the higher mass state $\nu_{3}$ and the lower mass doublet $\nu_{1,2}$, with $\nu_{3}$ being richer in $\nu_{\tau}$ or $\nu_{\mu}$ according to $\theta_{23}$ being in the first or second octant (while the $\nu_{1,2}$ doublet is always richer in $\nu_{e}$ ). If $F_{e}^{0}>F_{\mu, \tau}^{0}$, namely $E<E_{\text {eq }}$, then $\Delta m^{2}$-driven conversions are dominantly of the kind $\nu_{e} \rightarrow \nu_{\tau}$ $\left(\nu_{e} \rightarrow \nu_{\mu}\right)$ for $\sin ^{2} \theta_{23}<1 / 2\left(\sin ^{2} \theta_{23}>1 / 2\right)$, so that the final $\nu_{\tau}\left(\nu_{\mu}\right)$ flavor increases at the expenses of $\nu_{\mu}\left(\nu_{\tau}\right)$. The opposite happens if $F_{e}^{0}<F_{\mu, \tau}^{0}$, namely $E>E_{\text {eq }}$. The same reasoning hold for antineutrinos.

These arguments explain the main qualitative features of the numerical results in the lower panels of Fig. 5 which, by themselves, have just an academic interest. They may serve, however, as benchmarks in the exploration of extended SN scenarios with nonstandard initial conditions [37] or interactions [38] in the $\nu_{\mu}-\nu_{\tau}$ sector. 


\section{Final fluxes at the detector}

After collective effects have ended, and the $H$-resonance region is traversed, the flavor evolution is eventually subject to the so-called " $L$ resonance" at $\lambda \sim \omega_{L}$, which can be assumed adiabatic (see, e.g., [30]). [As noted, additional effects due to possible shock wave features or density fluctuations are ineffective for $\theta_{13}$ as low as in Eq. (17).]

In the specific context of self-interacting $\mathrm{SN}$ neutrinos in three families, final $L$ resonance effects have also been numerically verified in [17], by continuing the numerical evolution up to a few thousand $\mathrm{km}$. Here we do not repeat this numerical check of long-distance evolution, but take the resulting effects for granted; for completeness, we rephrase the related arguments of [17] in our notation as follows.

In the absence of self interactions effects, a strongly nonadiabatic $H$ resonance, plus an adiabatic $L$ resonance, would eventually distribute the original fluxes over the effective mass eigenstates $\nu_{i}$ and $\bar{\nu}_{i}$ as [30]:

$$
\begin{aligned}
& F_{1}=F_{x}^{0}, \\
& F_{2}=F_{e}^{0}, \\
& F_{3}=F_{x}^{0}, \\
& \bar{F}_{1}=\bar{F}_{e}^{0}, \\
& \bar{F}_{2}=\bar{F}_{x}^{0}, \\
& \bar{F}_{3}=\bar{F}_{x}^{0} .
\end{aligned}
$$

Spectral splits, however, alter these standard MSW expectations and swap the flavor contents in the effective $(e, 3)$ sector [17], so that

$$
\begin{aligned}
& F_{1}=F_{x}^{0}, \\
& F_{2}=F_{e}^{0} P_{e e}+F_{x}^{0}\left(1-P_{e e}\right), \\
& F_{3}=F_{x}^{0} P_{e e}+F_{e}^{0}\left(1-P_{e e}\right), \\
& \bar{F}_{1}=\bar{F}_{e}^{0} \bar{P}_{e e}+\bar{F}_{x}^{0}\left(1-\bar{P}_{e e}\right), \\
& \bar{F}_{2}=\bar{F}_{x}^{0}, \\
& \bar{F}_{3}=\bar{F}_{x}^{0} \bar{P}_{e e}+\bar{F}_{e}^{0}\left(1-\bar{P}_{e e}\right),
\end{aligned}
$$

where $P_{e e}$ and $\bar{P}_{e e}$, embedding collective effects, have a stepwise behavior as in Eqs. (1) and (2). The final, phase-averaged fluxes for the electron flavor at detection are given by

$$
\begin{aligned}
& F_{e}=\sum\left|U_{e i}\right|^{2} F_{i} \simeq \cos ^{2} \theta_{12} F_{1}+\sin ^{2} \theta_{12} F_{2}, \\
& \bar{F}_{e}=\sum\left|U_{e i}\right|^{2} \bar{F}_{i} \simeq \cos ^{2} \theta_{12} \bar{F}_{1}+\sin ^{2} \theta_{12} \bar{F}_{2},
\end{aligned}
$$

which, together with Eqs. (43)-(48), reproduce the limiting behaviors discussed in [17],

$$
\begin{aligned}
& F_{e} \simeq \begin{cases}\cos ^{2} \theta_{12} F_{x}^{0}+\sin ^{2} \theta_{12} F_{e}^{0} & \left(E<E_{c}\right), \\
F_{x}^{0} & \left(E>E_{c}\right),\end{cases} \\
& \bar{F}_{e} \simeq \begin{cases}\sin ^{2} \theta_{12} \bar{F}_{x}^{0}+\cos ^{2} \theta_{12} \bar{F}_{e}^{0} & \left(E<\bar{E}_{c}\right), \\
\bar{F}_{x}^{0} & \left(E>\bar{E}_{c}\right),\end{cases}
\end{aligned}
$$



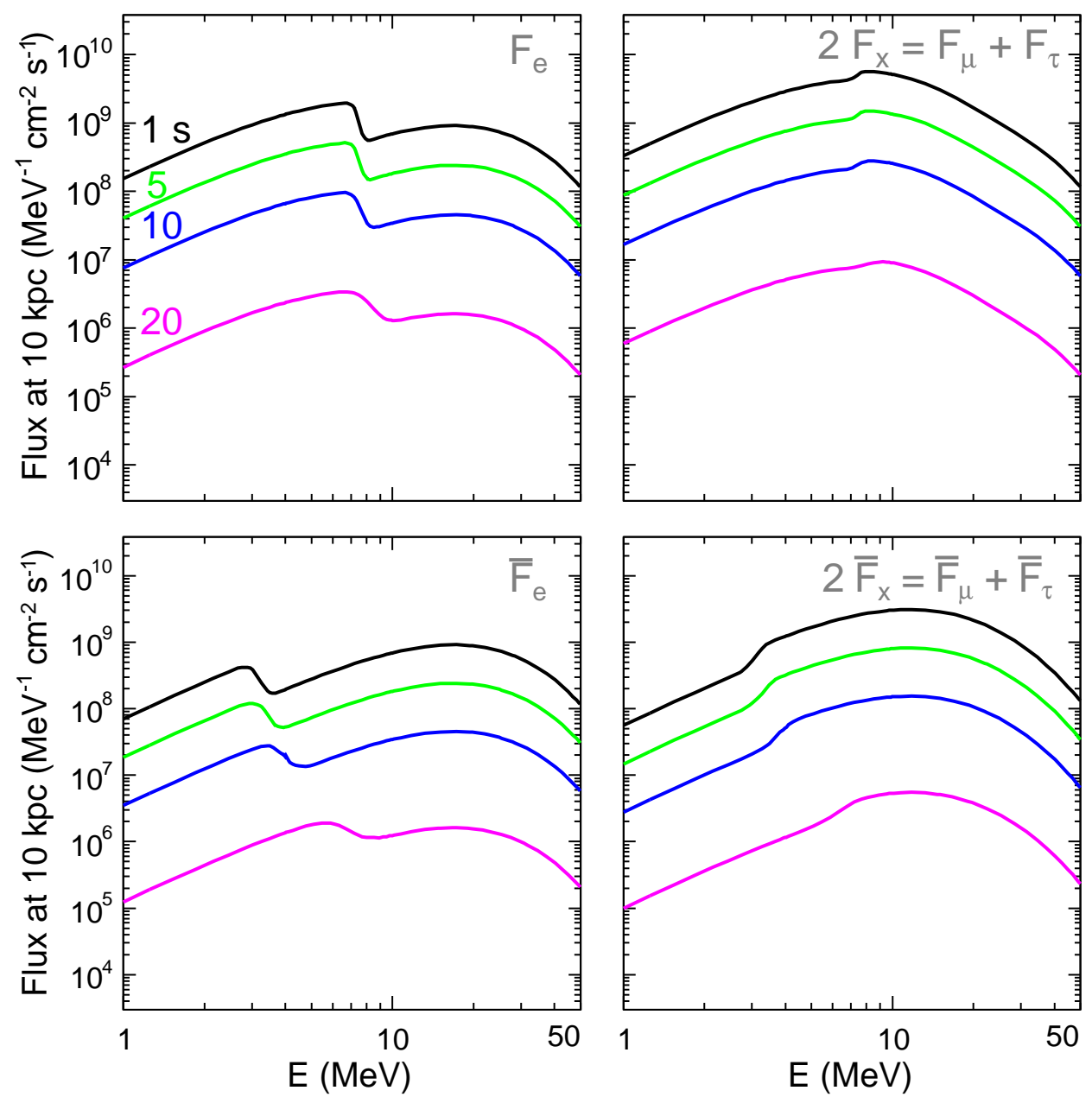

Figure 6. Final oscillated fluxes of neutrinos $\left(F_{\alpha}\right)$ and antineutrinos $\left(\bar{F}_{\alpha}\right)$ at $d=10 \mathrm{kpc}$. The comparison with the unoscillated fluxes in Fig. 1 shows the imprints of collective flavor evolution effects.

where the low-energy $\bar{\nu}$ split, not considered in [17], is also included.

By using Eqs. (6) -(91), one can eliminate $P_{e e}$ and $\bar{P}_{e e}$ in terms of the fluxes $F_{\alpha}^{\prime}$ at the end of collective effects. The final fluxes $F_{e}$ and $\bar{F}_{e}$ can thus be expressed in terms of the initial fluxes in Fig. 1 and of the intermediate computed fluxes in Fig. 3,

$$
\begin{aligned}
& F_{e}=\cos ^{2} \theta_{12} F_{x}^{0}+\sin ^{2} \theta_{12} F_{e}^{\prime}, \\
& \bar{F}_{e}=\cos ^{2} \theta_{12} \bar{F}_{e}^{\prime}+\sin ^{2} \theta_{12} \bar{F}_{x}^{0} .
\end{aligned}
$$

Finally, conservation of the total $\nu$ and $\bar{\nu}$ flux provides $F_{x}$ and $\bar{F}_{x}$ by subtraction,

$$
\begin{aligned}
& 2 F_{x}=\left(2 F_{x}^{0}+F_{e}^{0}\right)-F_{e}, \\
& 2 \bar{F}_{x}=\left(2 \bar{F}_{x}^{0}+\bar{F}_{e}^{0}\right)-\bar{F}_{e} .
\end{aligned}
$$

Figure 6 shows the corresponding results in graphical form. The spectral split features in the $\nu_{e}$ and $\bar{\nu}_{e}$ spectra are somewhat reduced by the low-energy $\theta_{12}$ mixing 
from Eqs. (49) and (50), but are still clearly observable at any time $t \geq 1 \mathrm{~s}$. The $x$-flavor split features are instead more suppressed by mixing.

We emphasize that, in view of prospective observations of galactic SN neutrino bursts (at least for the electron flavor via charged currents) the persistence of similar stepwise features for several seconds is rather useful. Detection of such features (if realized in nature) requires setting the threshold at low energy ( $\lesssim$ few $\mathrm{MeV}$ ): a very challenging goal, since one has to fight against backgrounds (which may be large in some shallow detector projects [39]) and low cross sections. However, low signal rates may be at least compensated by integration over time intervals of a few seconds, without canceling the persisting split effects. In order to facilitate feasibility studies in specific experimental settings, we provide our main results (i.e., the final fluxes at $d=10 \mathrm{kpc}$ in Fig. 6, as compared to the unoscillated ones in Fig. 1) in computer-readable form upon request.

\section{Effects of variations in the input SN neutrino spectra}

Our references choice of neutrino energy spectra and luminosities is by no means unique. Different choices may be motivated by the results of some SN explosion simulations, see e.g. [40]. We do not consider herein possible deviations from the hypothesis of luminosity equipartition among different neutrino species [40], which are not supported in our reference time interval $(t=1-20 \mathrm{~s})$ by the results of [26], and which might lead to new and more complicated split features 41 beyond the scope of this paper. However, we do study some interesting variations of the average energies $\left\langle E_{\alpha}\right\rangle$.

In particular, following Ref. [42] (inspired by [40]) one may adopt the values:

$$
\left\langle E_{e}\right\rangle=12 \mathrm{MeV},\left\langle E_{e}\right\rangle=15 \mathrm{MeV},\left\langle E_{x}\right\rangle=18 \mathrm{MeV} \text { (at any } t \text { ) , }
$$

which are much closer to each other than our reference choice in Eq. (22). We shall refer to the choice in Eq. (57) as to the "smaller $\Delta\langle E\rangle$ scenario."

One may also include average energies with noticeable changes with time, as suggested, e.g., by the results in [26], which read:

$$
\begin{aligned}
& \left\langle E_{e}\right\rangle=13 \mathrm{MeV},\left\langle E_{e}\right\rangle=16 \mathrm{MeV},\left\langle E_{x}\right\rangle=23 \mathrm{MeV}(t=1 \mathrm{~s}) ; \\
& \left\langle E_{e}\right\rangle=11 \mathrm{MeV},\left\langle E_{e}\right\rangle=18 \mathrm{MeV},\left\langle E_{x}\right\rangle=25 \mathrm{MeV}(t=5 \mathrm{~s}) ; \\
& \left\langle E_{e}\right\rangle=11 \mathrm{MeV},\left\langle E_{e}\right\rangle=20 \mathrm{MeV},\left\langle E_{x}\right\rangle=25 \mathrm{MeV}(t=10 \mathrm{~s}) ; \\
& \left\langle E_{e}\right\rangle=11 \mathrm{MeV},\left\langle E_{e}\right\rangle=20 \mathrm{MeV},\left\langle E_{x}\right\rangle=25 \mathrm{MeV}(t=20 \mathrm{~s}) .
\end{aligned}
$$

We shall refer to such assignments as to the "time-dependent $\langle E\rangle$ scenario."

For fixed energy luminosity [taken as in Eq. (19)], variations in the average neutrino energies lead to variations in their total number and thus also in the self-interaction potential $\mu$, as compared to the reference one shown in Fig. 2. Figure 7 shows the radial profile of $\mu$ for the case of smaller $\Delta\langle E\rangle$ (left panel) and of time-dependent $\langle E\rangle$ (right panel). The shaded bands corresponds to the range $\mu \in\left[\mu_{\text {inf }}, \mu_{\text {sup }}\right]$ where, 

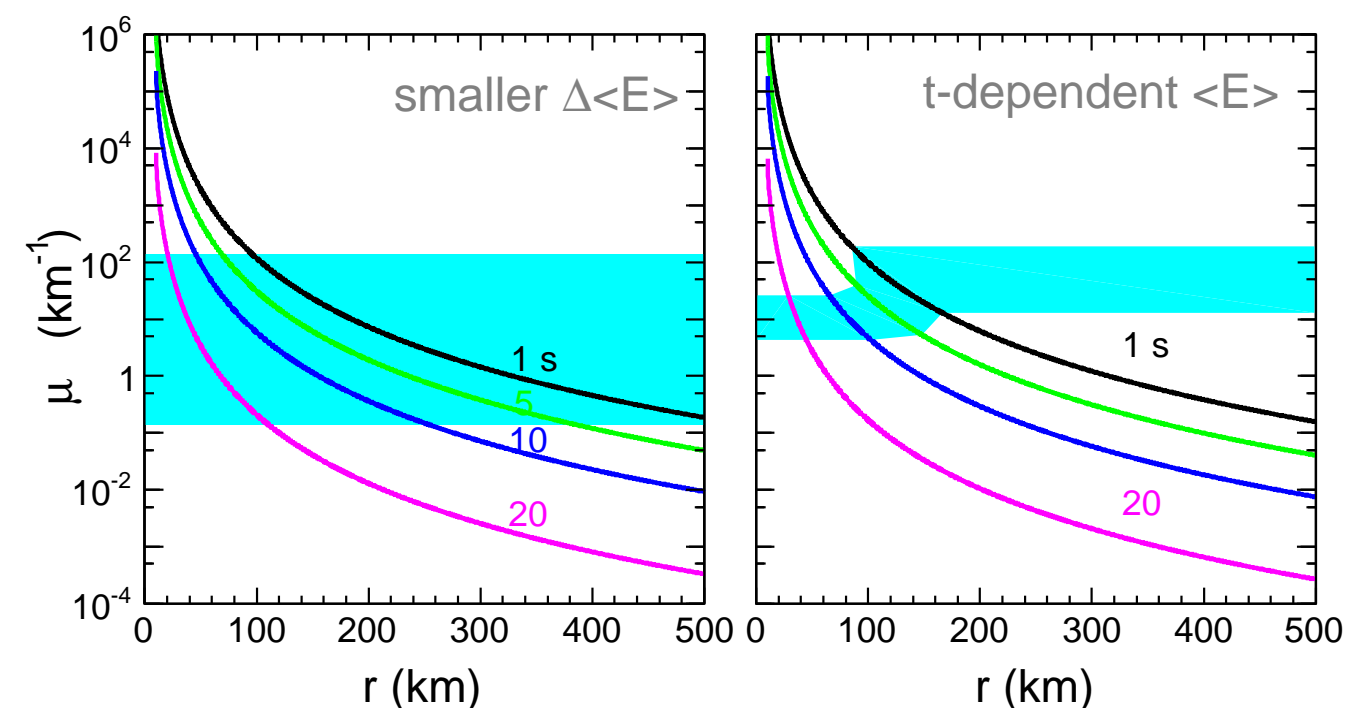

Figure 7. Radial profiles of the self-interaction potential $\mu$ at $t=1,5,10$, and $20 \mathrm{~s}$ for two different variations of the supernova neutrino input (as compared with Fig. 2). Left: case with smaller differences among (constant) average energies. Right: case with time-dependent average energies. The shaded bands mark the $\mu$ range where bipolar effects develop. The band acquires a time dependence in the right panel. See the text for details.

according to the discussion in [10], collective bipolar oscillations occur; the range is timedependent in the right panel. Both the $\mu(r)$ curves and the bands in Fig. 7 differ from the corresponding ones in Fig. 2. In particular, at $t=20 \mathrm{~s}$, the intersection of the $\mu(r)$ curve with the shaded band in Fig. 7 leads to the smallest radial interval $(\Delta r \simeq 15 \mathrm{~km})$ for the development of bipolar oscillations - a fact that leads to an interesting consequence, as shown below.

Figure 8 shows the results of the neutrino flavor evolution (at the end of collective effects) in the case of smaller $\Delta\langle E\rangle$. Compared with our reference scenario in Fig. 3, the spectral split features are qualitatively similar but less pronounced, as expected from the fact the spectral differences of different species are smaller. In particular, the step-like variation across the split is reduced by a factor of two or more, for both neutrinos (around $E_{c} \simeq 7.5 \mathrm{MeV}$ ) and antineutrinos. From the experimental viewpoint, the smaller the split variations, the higher the energy resolution and the statistics needed to observe them. Future SN explosion simulations will shed new light on the expected (dis)similarities among the unoscillated spectra of $\nu_{e}, \bar{\nu}_{e}$ and $\nu_{x}$, and thus on the size of observable oscillation effects (collective or not).

Figure 9 is analogous to Fig. 8, but refers to the case of time-dependent $\langle E\rangle$ (to be compared with the reference scenario in Fig. 3). In this case, the critical split energies appear to be also time-dependent. For neutrinos, conservation of net lepton number 


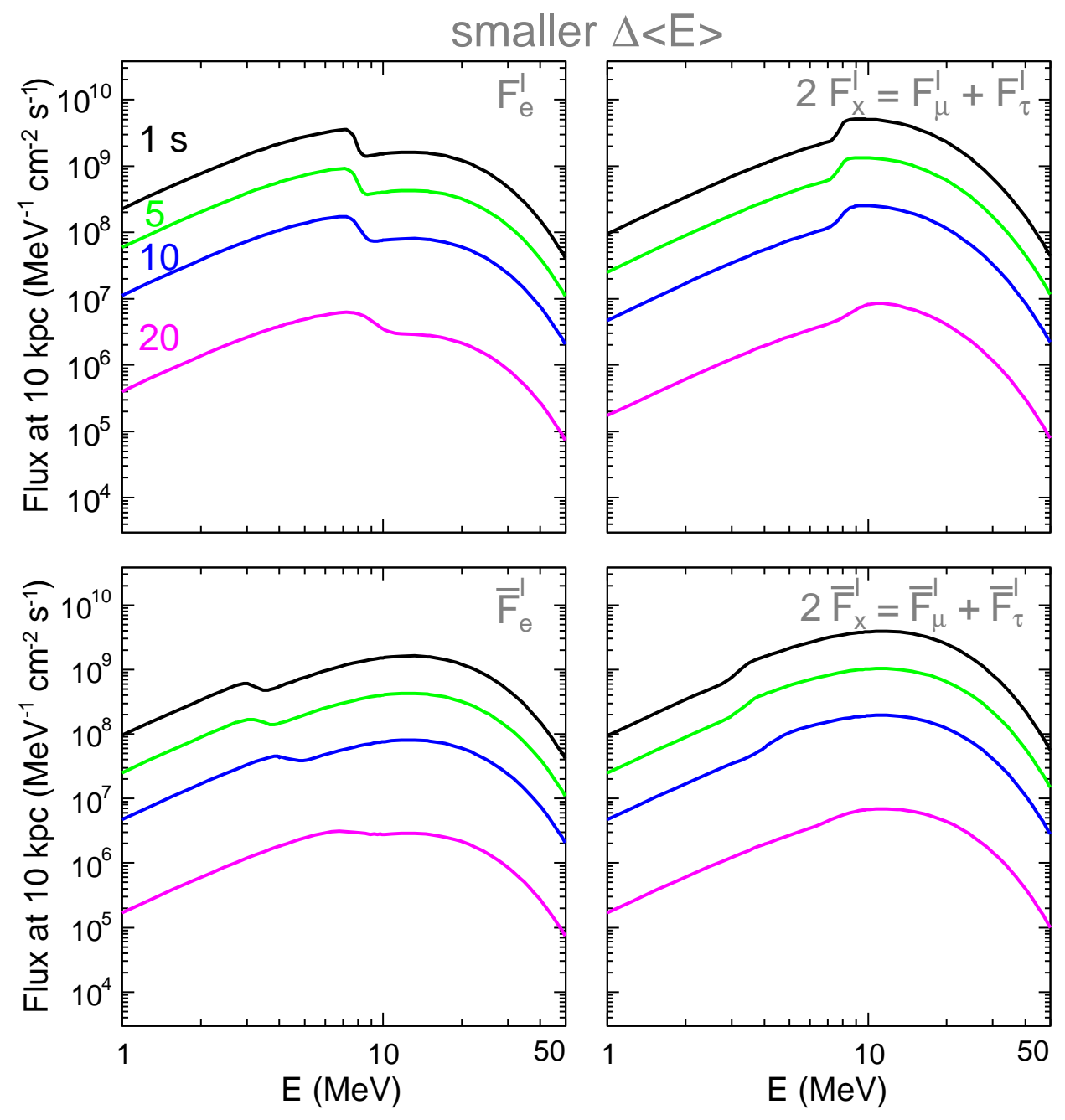

Figure 8. Fluxes of neutrinos $\left(F_{\alpha}^{\prime}\right)$ and antineutrinos $\left(\bar{F}_{\alpha}^{\prime}\right)$ at the end of collective effects, for the case with smaller $\Delta\langle E\rangle$ (as compared with the reference scenario in Fig. 3).

(assuming no $\bar{\nu}$ split in first approximation) predicts $E_{c} \simeq 7.2,8.9,9.7$ and $9.7 \mathrm{MeV}$ for the chosen spectra at $t=1,5,10$ and $20 \mathrm{~s}$. This trend is consistent with the numerical results in the upper panels of Fig. 9. Antineutrinos (lower panels) also show a steadily increasing split energy, which we are unable to estimate a priori, however. The increase in critical energies with time may favorable from the experimental viewpoint, since the cross section increase compensate in part the luminosity decrease; on the other hand, it may prevent integration of spectra on time intervals larger than $\Delta t \sim 1 \mathrm{~s}$, where the split feature would be blurred.

Interestingly, no split appear in Fig. 9 at $t=20 \mathrm{~s}$ : the spectra are basically unoscillated. This fact is related to the very short range $(\Delta r \simeq 16 \mathrm{~km})$ expected for the development of bipolar oscillations at $t=20 \mathrm{~s}$, as noted above. It turns out that 


\section{t-dependent $<$ E>}
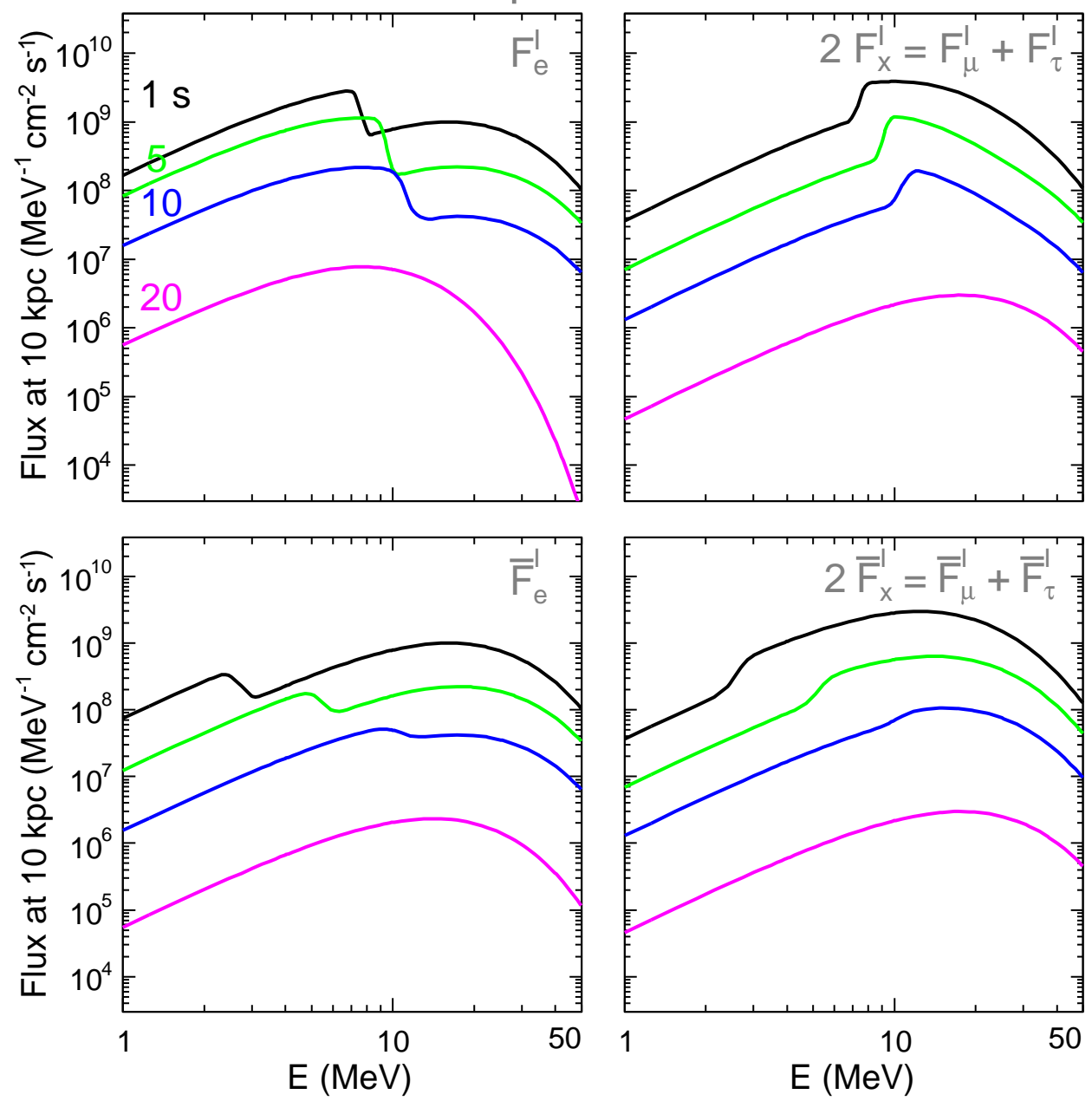

Figure 9. Fluxes of neutrinos $\left(F_{\alpha}^{\prime}\right)$ and antineutrinos $\left(\bar{F}_{\alpha}^{\prime}\right)$ at the end of collective effects, for the case with time-dependent $\langle E\rangle$ (as compared with the reference scenario in Fig. 3).

the period of bipolar oscillations is of the same order of $\Delta r$ in this case and, literally, the "flavor pendulum" has not enough time to make a single swing: it remains frozen in the upward, unstable equilibrium position, due to the extremely sudden decrease of $\mu(r)$. Conversely, in our reference scenario at $t=20 \mathrm{~s}$ (see Fig. 3) the range $\Delta r$ happens to be slightly larger, thus enabling the flavor pendulum to make a full swing and to start the collective transitions, which eventually lead to the spectral split. At late time, the neutrino and antineutrino splits appears thus to be relatively fragile phenomena, whose absence or presence might provide, in principle, some information about the gradient of the self-interaction neutrino potential $\mu(r)$. 


\section{Summary}

Building upon recent literature on $3 \nu$ collective effects in core-collapse supernovae [15, 16, 17, 18, 19, 20, 21, 22, we have performed an independent study of three-flavor evolution of neutrinos and antineutrinos within a rather "standard" SN model (Figs. 1 and 2). Then, assuming inverted neutrino mass hierarchy and tiny $\theta_{13}$ (i.e., strongly nonadiabatic $H$-resonance), self-interaction effects are expected to provide the dominant spectral features, in the form of "splits" — or "stepwise swaps" — for both $\nu$ and $\bar{\nu}$.

We have explored this SN $3 \nu$ scenario by solving the evolution equations for the $3 \nu$ density matrix in vector form and single-angle approximation, after discretization in energy. The numerical results for the evolved fluxes at the end of collective effects (Fig. 3) confirm basic expectations at low and high energy [Eqs. (6) - (9)] in terms of unevolved fluxes (Fig. 4). Effects of the "solar" mass splitting and of the $\nu_{\mu}-\nu_{\tau}$ interaction energy difference in matter are found to be negligible; effects due to variations of $\theta_{23}$ are large (Fig. 5) but unobservable. The final fluxes at the detector (Fig. 6) are obtained by standard application of adiabatic $L$-resonance effects.

Both $\nu$ and $\bar{\nu}$ spectral split features tipically emerge with similar characteristics in the whole time interval considered $(t=1-20 \mathrm{~s})$. Observations of such features (if realized in nature) and of their possible variations require low-energy thresholds, where the signal is suppressed by low cross sections; however, time integration can partly overcome the suppression, without necessarily canceling the persistent spectral features. If the collected statistics and the energy resolution allow, spectra collected at different times might even reveal migrations of the split energies due to time variations of neutrino temperatures. The low-energy frontier in SN neutrino physics may thus be the key to access the physics of inverted mass hierarchy, especially if $\theta_{13}$ is very small.

\section{Acknowledgments}

This work is supported in part by the Italian "Istituto Nazionale di Fisica Nucleare" (INFN) and "Ministero dell'Istruzione, dell'Università e della Ricerca" (MIUR) through the "Astroparticle Physics" research project. I.T. acknowledges support by the E.U. (ENTApP network) at the Fifth Annual ENTApP meeting "Neutrinos in Particle, in Nuclear, and in Astro-Physics" (ECT*, Trento, Italy, 2008), where preliminary results of this work were presented. We thank A. Mirizzi for careful reading of the manuscript and for very useful remarks and suggestions. 
Supernova neutrino three-flavor evolution with dominant collective effects

\section{References}

[1] A. Dighe, "Physics potential of future supernova neutrino observations," Proceedings of Neutrino 2008, 23rd International Conference on Neutrino Physics and Astrophysics (Christchurch, New Zealand, 2008) arXiv:0809.2977 [hep-ph].

[2] H. T. Y. Janka, K. Langanke, A. Marek, G. Martinez-Pinedo and B. Mueller, "Theory of CoreCollapse Supernovae," Phys. Rept. 442, 38 (2007) arXiv:astro-ph/0612072.

[3] H. Duan, G. M. Fuller and Y. Z. Qian, "Collective Neutrino Flavor Transformation In Supernovae," Phys. Rev. D 74, 123004 (2006) arXiv:astro-ph/0511275.

[4] H. Duan, G. M. Fuller, J. Carlson and Y. Z. Qian, "Simulation of coherent non-linear neutrino flavor transformation in the supernova environment. I: Correlated neutrino trajectories," Phys. Rev. D 74, 105014 (2006) arXiv:astro-ph/0606616.

[5] J. T. Pantaleone, "Neutrino oscillations at high densities," Phys. Lett. B 287, 128 (1992).

[6] S. Hannestad, G. G. Raffelt, G. Sigl and Y. Y. Y. Wong, "Self-induced conversion in dense neutrino gases: Pendulum in flavour space," Phys. Rev. D 74, 105010 (2006) [Erratum-ibid. D 76, 029901 (2007)] arXiv:astro-ph/0608695.

[7] H. Duan, G. M. Fuller and J. Carlson, "Simulating nonlinear neutrino flavor evolution," arXiv:0803.3650 [astro-ph].

[8] G. G. Raffelt and A. Y. Smirnov, "Self-induced spectral splits in supernova neutrino fluxes," Phys. Rev. D 76, 081301 (2007) [Erratum-ibid. D 77, 029903 (2008)] [arXiv:0705.1830 [hep-ph]].

[9] H. Duan, G. M. Fuller, J. Carlson and Y. Q. Zhong, "Neutrino Mass Hierarchy and Stepwise Spectral Swapping of Supernova Neutrino Flavors," Phys. Rev. Lett. 99, 241802 (2007) arXiv:0707.0290 [astro-ph]].

[10] G. L. Fogli, E. Lisi, A. Marrone and A. Mirizzi, "Collective neutrino flavor transitions in supernovae and the role of trajectory averaging," JCAP 0712, 010 (2007) arXiv:0707.1998 [hep-ph]].

[11] G. L. Fogli, E. Lisi, A. Marrone, A. Mirizzi and I. Tamborra, "Low-energy spectral features of supernova (anti)neutrinos in inverted hierarchy," Phys. Rev. D 78, 097301 (2008) arXiv:0808.0807 [hep-ph]].

[12] H. Duan, G. M. Fuller, J. Carlson and Y. Z. Qian, "Analysis of Collective Neutrino Flavor Transformation in Supernovae," Phys. Rev. D 75, 125005 (2007) arXiv:astro-ph/0703776.

[13] H. Duan, G. M. Fuller and Y. Z. Qian, "A Simple Picture for Neutrino Flavor Transformation in Supernovae," Phys. Rev. D 76, 085013 (2007) arXiv:0706.4293 [astro-ph]].

[14] G. G. Raffelt and A. Y. Smirnov, "Adiabaticity and spectral splits in collective neutrino transformations," Phys. Rev. D 76, 125008 (2007) [arXiv:0709.4641 [hep-ph]].

[15] H. Duan, G. M. Fuller, J. Carlson and Y. Z. Qian, "Flavor Evolution of the Neutronization Neutrino Burst from an O-Ne-Mg Core-Collapse Supernova," Phys. Rev. Lett. 100, 021101 (2008) arXiv:0710.1271 [astro-ph]].

[16] A. Esteban-Pretel, S. Pastor, R. Tomas, G. G. Raffelt and G. Sigl, "Mu-tau neutrino refraction and collective three-flavor transformations in supernovae," Phys. Rev. D 77, 065024 (2008) arXiv:0712.1137 [astro-ph]].

[17] B. Dasgupta and A. Dighe, "Collective three-flavor oscillations of supernova neutrinos," Phys. Rev. D 77, 113002 (2008) arXiv:0712.3798 [hep-ph]].

[18] H. Duan, G. M. Fuller and Y. Z. Qian, "Stepwise Spectral Swapping with Three Neutrino Flavors," Phys. Rev. D 77, 085016 (2008) [arXiv:0801.1363 [hep-ph]].

[19] B. Dasgupta, A. Dighe, A. Mirizzi and G. G. Raffelt, "Spectral split in prompt supernova neutrino burst: Analytic three-flavor treatment," Phys. Rev. D 77, 113007 (2008) arXiv:0801.1660 [hep$\mathrm{ph}]$.

[20] B. Dasgupta, A. Dighe and A. Mirizzi, "Identifying neutrino mass hierarchy at extremely small theta(13) through Earth matter effects in a supernova signal," Phys. Rev. Lett. 101, 171801 (2008) arXiv:0802.1481 [hep-ph]].

[21] S. Chakraborty, S. Choubey, B. Dasgupta and K. Kar, "Effect of Collective Flavor Oscillations 
on the Diffuse Supernova Neutrino Background," JCAP 0809, 013 (2008) arXiv:0805.3131 [hep-ph]].

[22] J. Gava and C. Volpe, "Collective neutrinos oscillation in matter and CP-violation," Phys. Rev. D 78, 083007 (2008) arXiv:0807.3418 [astro-ph]].

[23] C. Amsler et al. [Particle Data Group], "Review of particle physics," Phys. Lett. B 667, 1 (2008).

[24] G. L. Fogli et al., "Observables sensitive to absolute neutrino masses (Addendum)," Phys. Rev. D 78, 033010 (2008) arXiv:0805.2517 [hep-ph]].

[25] R. C. Schirato and G. M. Fuller "Connection between supernova shocks, flavor transformation, and the neutrino signal," arXiv:astro-ph/0205390.

[26] T. Totani, K. Sato, H. E. Dalhed and J. R. Wilson, "Future detection of supernova neutrino burst and explosion mechanism," Astrophys. J. 496, 216 (1998) arXiv:astro-ph/9710203.

[27] H. Duan, G. M. Fuller, J. Carlson and Y. Z. Qian, "Analysis of Collective Neutrino Flavor Transformation in Supernovae," Phys. Rev. D 75, 125005 (2007) arXiv:astro-ph/0703776.

[28] S. Pastor, G. G. Raffelt and D. V. Semikoz, "Physics of synchronized neutrino oscillations caused by self-interactions," Phys. Rev. D 65, 053011 (2002) arXiv:hep-ph/0109035].

[29] L. Wolfenstein, "Neutrino Oscillations In Matter," Phys. Rev. D 17, 2369 (1978); S. P. Mikheev and A. Yu. Smirnov, "Resonance Enhancement Of Oscillations In Matter And Solar Neutrino Spectroscopy," Yad. Fiz. 42, 1441 (1985) [Sov. J. Nucl. Phys. 42, 913 (1985)].

[30] A. S. Dighe and A. Y. Smirnov, "Identifying the neutrino mass spectrum from the neutrino burst from a supernova," Phys. Rev. D 62, 033007 (2000) arXiv:hep-ph/9907423.

[31] G. L. Fogli, E. Lisi, A. Mirizzi and D. Montanino, "Probing supernova shock waves and neutrino flavor transitions in next-generation water-Cherenkov detectors," JCAP 0504, 002 (2005) arXiv:hep-ph/0412046.

[32] G. L. Fogli, E. Lisi, A. Mirizzi and D. Montanino, "Damping of supernova neutrino transitions in stochastic shock-wave density profiles," JCAP 0606, 012 (2006) arXiv:hep-ph/0603033.

[33] A. Esteban-Pretel, A. Mirizzi, S. Pastor, R. Tomas, G. G. Raffelt, P. D. Serpico and G. Sigl, "Role of dense matter in collective supernova neutrino transformations," Phys. Rev. D 78, 085012 (2008) arXiv:0807.0659 [astro-ph]].

[34] Irene Tamborra, Master Thesis (Tesi di Laurea, in Italian): "Auto-interazioni ed oscillazioni di neutrini in una supernova con collasso del nucleo" (U. of Bari, October 2007).

[35] F.J. Botella, C.S. Lim and W.J. Marciano, "Radiative corrections to neutrino indices of refraction," Phys. Rev. D 35, 896 (1987).

[36] A. Esteban-Pretel, S. Pastor, R. Tomas, G. G. Raffelt and G. Sigl, "Decoherence in supernova neutrino transformations suppressed by deleptonization," Phys. Rev. D 76, 125018 (2007).

[37] E. K. Akhmedov, C. Lunardini and A. Y. Smirnov, "Supernova neutrinos: Difference of $\nu_{\mu}-\nu_{\tau}$ fluxes and conversion effects," Nucl. Phys. B 643, 339 (2002) arXiv:hep-ph/0204091.

[38] M. Blennow, A. Mirizzi and P. D. Serpico, "Nonstandard neutrino-neutrino refractive effects in dense neutrino gases," arXiv:0810.2297 [hep-ph].

[39] M. D. Kistler, H. Yuksel, S. Ando, J. F. Beacom and Y. Suzuki, "Core-Collapse Astrophysics with a Five-Megaton Neutrino Detector," arXiv:0810.1959 [astro-ph].

[40] R. Buras, H. T. Janka, M. T. Keil, G. G. Raffelt and M. Rampp, "Electron-neutrino pair annihilation: A new source for muon and tau neutrinos in supernovae," Astrophys. J. 587, 320 (2003) arXiv:astro-ph/0205006.

[41] B. Dasgupta, talk at Workshop "Frontiers in theoretical neutrino physics" (Paris, France, 16-19 March 2009). Website: ipnweb.in2p3.fr/ frontiers

[42] R. Tomas, M. Kachelriess, G. Raffelt, A. Dighe, H. T. Janka and L. Scheck, "Neutrino signatures of supernova shock and reverse shock propagation," JCAP 0409, 015 (2004) arXiv:astro-ph/0407132. 